\title{
ABORDAJE DE ENFERMERÍA SOBRE EL MIEDO Y ANSIEDAD ANTE LA MUERTE EN PERSONAS ADULTAS MAYORES
}

\author{
NURSING APPROACH TO FEAR AND ANXIETY ABOUT DEATH IN OLDER ADULTS \\ Idalia M. Herrera, Isaí A. Medina, Ana L. Carrillo Y Diana B. Montelongo \\ Universidad Autónoma de Coahuila (México)
}

\begin{abstract}
Ante los marcados cambios poblacionales que indican una elevación en la concentración de personas adultas mayores, es relevante visualizar un panorama de atención en salud integral tomando en cuenta las preocupaciones y emociones que se pueden presentar en la etapa de la vejez por lo cual es necesario tomar en cuenta el abordar temáticas como el fin de la existencia o la muerte que es una parte natural de la condición humana. Aunque los aspectos sociales, religiosos, culturales, psicológicos, ambientales, políticos y económicos direccionan la mayoría de las veces la percepción o idea que tenga el sujeto acerca de la muerte, las emociones que regularmente se encuentran asociadas ante tal hecho inminente son el miedo y ansiedad los cuales repercuten en el estado de salud de las personas mayores disminuyendo su bienestar físico y emocional y a la vez le impide concientizar o reflexionar acerca de los logros personales alcanzados, nuevas metas de vida y reconocimiento de su esencia y valor humano. Es por ello que el reflexionar acerca de las intervenciones clave de enfermería que pueden mitigar o disminuir este tipo de temores o respuestas ante la muerte marcará el camino para emprender acción y brindar atención de calidad.
\end{abstract}

Palabras clave: miedo, ansiedad, muerte, enfermería geriátrica.

Given the marked population changes that indicate an increase in the concentration of older adults, It is relevant to visualize a comprehensive health care panorama taking into account the concerns and emotions that may arise in the old age stage so it is necessary to take into account the approach to topics such as the end of existence or death that is a part of the human condition. Although the social, religious, cultural, psychological, environmental, political, and economic aspects most of the time address the subject's perception or idea of death, the emotions that are regularly associated with such an imminent event are fear and anxiety which have an impact on the health status of older people by decreasing their physical and emotional well-being and the same time prevents them from raising awareness or reflecting on the achievements individuals reached, new goals of life and recognition of its essence and human value. That's why thinking about key nursing interventions that can mitigate or lessen these kinds of fears or responses to death will set the stage for action and quality care.

Keywords: fear, anxiety, death, geriatric nursing.

Las evidentes modificaciones de los índices demográficos hacen referencia particularmente a un incremento poblacional, estableciendo un énfasis en el grupo de la tercera edad donde se

Idalia Margarita Herrera Garibay, Facultad de Enfermería "Dr. Santiago Valdés Galindo". Universidad Autónoma de Coahuila, México, https://orcid.org/0000-0002-6012-7033

Isaí Arturo Medina Fernández, Facultad de Enfermería “Dr. Santiago Valdés Galindo”. Universidad Autónoma de Coahuila, México, https://orcid.org/0000-0003-2845-4648

Ana Laura Carrillo Cervantes, Facultad de Enfermería “Dr. Santiago Valdés Galindo". Universidad Autónoma de Coahuila, México, correspondencia alcc 73@hotmail.com, https://orcid.org/0000-0003-2920-4675

Diana Berenice Montelongo Cortés, Facultad de Enfermería “Dr. Santiago Valdés Galindo". Universidad Autónoma de Coahuila, México, https://orcid.org/0000-0003-4254-2468 
espera que para el año 2050 se duplique la prevalencia de personas adultas mayores (PAM) pasando del $12 \%$ al 22\% (OMS, 2018). A estas implicaciones demográficas se le suman cambios epidemiológicos y sociales que en su conjunto favorecen el envejecimiento poblacional lo cual guarda estrecha relación con la necesidad de que se plantee una visión futura y una posterior preparación para atender los diversos retos, compromisos y realidades a los que se enfrentaran los sectores de atención en materia de salud (Ochoa, Cruz, Pérez y Cuevas, 2018).

El envejecimiento es un proceso natural de los seres vivos, el cual se refiere al conjunto de transformaciones que se presentan a nivel sistémico-biológico (cambios progresivos en las células, tejidos, órganos, etc.) a consecuencia del paso del tiempo, éste comienza con la concepción y termina con la muerte (Ceberio, 2013). En este aspecto, es cierto que los avances tecnológicos, el acceso a los servicios médicos, las nuevas investigaciones y el incremento de la esperanza de vida se han visto presentes a favor del individuo, sin embargo, se debe tener muy en cuenta que el fin de la existencia o la muerte es una parte natural de la condición humana de la que nadie está exento (Sugar, 2019).

La muerte es reconocida como un hecho adherido a la vida del ser humano y ha sido objeto de cuestionamientos, incertidumbres y reflexiones en el individuo. Relacionada a connotaciones religiosas y culturales, es sin duda alguna, un fenómeno de gran importancia en la existencia del hombre (Meza, 2011). En situaciones donde se es consciente sobre la proximidad de la culminación de la vida se pueden presentar respuestas e inestabilidades emocionales tales como ansiedad y miedo que en ocasiones pueden afectar directamente las relaciones familiares, estilos y calidad de vida (Uribe, Valderrama y López, 2007).

El miedo a la muerte según Villa (2011), concierne a un acontecimiento universal y hasta cierto punto natural, derivado principalmente del deseo de supervivencia de la persona, llevándolo a experimentar temor e incertidumbre sobre la manera en que morirá y que sucederá después. Este autor también refiere que la magnitud de tal estado desagradable se verá influenciada por el entorno cultural, sociohistórico y religioso. El miedo se presenta ante un enfoque especifico, no es constante y solo se verá reflejado cuando el individuo se encuentre en una situación de peligro percibida centrándose en aspectos concretos como la causa que pudiera ocasionar su muerte o al proceso de morir en sí (Tomás-Sábado, 2016).

Por otra parte, la ansiedad ante la muerte hace referencia a una tensión anticipada con base a una emoción intrínseca que genera inquietud, temor, inseguridad ante una situación de riesgo inespecífica que generalmente tiene relación con manifestaciones fisiológicas como temblor, fatiga, diaforesis y cifras alteradas de la frecuencia cardiaca y respiratoria (Navas \& Vargas, 2012). Propiamente, la North American Nursing Diagnosis Association (NANADA, 2018) dentro de sus etiquetas diagnósticas la define como la emoción ambigua de características inquietantes, preocupantes y temerosas respecto a los procesos anticipatorios de daño o finitud de la vida, refiriendo que la población susceptible asociada a este concepto incluye las personas con experiencias cercanas a la muerte, en procesos de enfermedad terminal y personas que se han creado cuestionamientos sobre el proceso de morir.

En definitiva, la preocupación sobre la muerte propia y ajena en las PAM les genera incertidumbre en el aspecto de querer saber qué es lo que sucede después de la misma y con regularidad se ve asociada a connotaciones negativas, sentimientos y actitudes como la tristeza, ansiedad, dolor, pérdida, miedo, enfermedades y sufrimiento, percepción que se basa en la diversidad del ser humano en cuanto a su formación, vivencias y experiencias (Tomás-Sábado, 
2016). También puede darse el caso de que, ante alguna enfermedad agregada en la vejez, la muerte sea vista como un escape o como una parte natural del ciclo vital esto cuando las PAM han logrado disfrutar plenamente su vida (Sugar, 2019).

El abordar el tema de la muerte en las PAM por parte del profesional de enfermería, es de gran relevancia pues da pie a establecer estrategias de atención y cuidado directamente en este grupo de edad que se ve afectado emocionalmente, en su construcción interna y de propósito de vida lo cual repercute en los aspectos familiares y sociales (Duran et al., 2020). Esto involucra manifestar el hecho de que es un fenómeno natural permanente y esencial a las circunstancias humanas donde se producen posiblemente más emociones (Tomás-Sábado, 2016).

Es así que, ante las respuestas emocionales de temor y ansiedad que experimentan las PAM al pensar sobre la cercanía del final de su vida, hace necesario un liderazgo por parte de los profesionales de la salud caracterizado por la implementación de competencias dirigidas hacia un acompañamiento integral tanto a los pacientes como a la familia (Meza, 2011). La intervención del profesional de enfermería implica incluir actividades que generen un avance en el entendimiento del proceso de muerte y que optimicen el sentido de vida, lo cual permitirá superar los miedos injustificados, logrando a su vez una vida digna, serena y responsable del autocuidado (Morales, Quintero y Pérez, 2011).

Por otra parte, desde el punto de vista metodológico se realizó una revisión de literatura de artículos, tesis y libros, destacando a su vez las contribuciones en el campo de enfermería. El objetivo de este artículo es reflexionar sobre las intervenciones de enfermería que aborden el miedo y ansiedad ante la muerte en Personas Adultas Mayores.

\section{La muerte en las PAM}

La muerte como tema central es definida como un evento natural que se distingue por la culminación de los mecanismos orgánicos y el cese de la actividad vital (función respiratoria, cardíaca y cerebral) que otorga soporte a la vida alejando al sujeto del plano existencial (Sánchez \& Salas, 2015). Se refiere a un proceso normal de todos los seres vivos que es definitivo y ocurrirá con seguridad pues no está aislado de la del contexto de la existencia (Cartay, 2002). Aunque la imagen de la muerte varía según la cultura y el momento histórico en el que se desarrolla la persona, regularmente es vista con temor y un problema que debe ser evitado por todos los medios posibles (Kübler-Ross, 2009).

Las concepciones de la muerte en las PAM enmarcan diferentes aprehensiones pues suelen cuestionarse acerca de la manera, el tiempo, el motivo y las circunstancias en que morirán cargando preocupaciones al imaginarse muriendo solos (as), presentar enfermedad que conlleve discapacidad y dolor o convertirse en una carga de cuidado y gasto monetario para sus familiares (Sugar, 2019). Por otro lado, cuando las PAM piensan en el final de su vida los lleva a una reflexión del tiempo transcurrido y desprendimiento de lo material, sin embargo, la vejez implica atravesar por pérdidas y muerte de seres allegados lo cual puede generar respuestas negativas o de serenidad, según el concepto que tenga la persona de la muerte y su grado de expresión emocional (Ceberio, 2013).

Precisamente, a medida que los individuos avanzan en edad, el miedo a la muerte puede estar causado, por ejemplo, por las falsas concepciones religiosas asociadas a castigo y condena aspectos aprendidos desde la niñez, por los apegos emocionales hacia los seres queridos y/o 
posesiones materiales o simplemente por miedo al sufrimiento y a lo desconocido. En este sentido, el miedo es expresado como una estrategia de supervivencia que ocurre cuando la persona se siente amenazada físicamente, posicionándolo en un estado de alerta, de ataque o de huida ante algo percibido como un peligro. En realidad, es un reflejo automático del inconsciente (Carmelo \& Comas, 2014).

Sobre la ansiedad ante la muerte, se trata de una reacción emocional que se origina debido a una serie de estímulos alrededor del individuo, los cuales pueden ser ambientales relacionado a funerales, cementerios o cadáveres, estímulos situacionales en relación a las experiencias que predisponen a la persona a reaccionar con temor, así como estímulos internos referentes a las ideas personales centradas en el deceso propio y de sus semejantes (Limonero, citado en TomásSábado, 2016).

Bajo un contexto de perdida de la salud importante, el miedo y ansiedad ante la muerte se refiere a las principales emociones que experimenta el enfermo frente a la muerte con un enfoque direccionado a la incertidumbre de que es lo que sucede en el camino del morir. Estas emociones se desprenden por el temor de perder a los seres queridos y a la soledad, por el miedo de perder el propio cuerpo, imagen, integridad e identidad, así como por el temor de perder el autocontrol y no conseguir un verdadero sentido de vida (Sandrin, 2013).

En efecto, el miedo y ansiedad ante la muerte son reconocidos como parte de las manifestaciones y respuestas de los individuos ante el fin de la vida e implica considerar múltiples aspectos a su alrededor, como por ejemplo los de tipo cultural, religioso, espiritual, familiar, personal, psicológico, ambiental, político y económico puesto que en ocasiones la persona se enraíza en ese temor como una estrategia de conservación para tratar de evitar la muerte y por tal miedo a morir deja de vivir el presente y se centra en una percepción dificultosa del futuro (Carmelo \& Comas 2014).

La muerte como detonante de miedo y ansiedad abordada en diferentes aportaciones de investigación, deja de manifiesto los diversos factores que también pueden intervenir o asociarse ante tal fenómeno. Lo anterior puede ser observado en el estudio realizado por Uribe et, al. (2007) en adultos mayores, el cual reflejó una mayor incidencia de "actitud de escape" respecto a la muerte y con el indicio de que las mujeres adultas mayores presentan más miedo hacia el tema de la muerte. Por otro lado, Álvarez y Flórez (2008) encontraron que los adultos mayores con enfermedad crónica (diabetes, hipertensión y cáncer) presentaron en su mayoría actitudes de aceptación de acercamiento hacia su propia muerte.

El miedo a la muerte también ha sido analizado incluyendo aspectos espirituales y religiosos de las PAM, como en la investigación de Restrepo (2013) quien pudo evidenciar que las personas experimentaban un mayor miedo a la muerte de otros a medida que incrementaban las prácticas y experiencias religiosas y expone a su vez que conforme aumenta este temor peor es la salud de los sujetos.

De igual manera, Brudek y Sekowski (2019) mostraron en su estudio, que la aceptación del enfoque de la muerte puede estar presente principalmente en personas con religiosidad inherente a la identidad del sujeto. Desde otra perspectiva, Rivarola (2016) concluye en que los aspectos espirituales pueden fungir como factores protectores ante el miedo a la propia muerte que experimentan las PAM pues aporta motivación y cierta estabilidad emocional. 
Además, los estudios sobre esta temática han incluido variables de carácter psicológico y de personalidad como la inteligencia emocional, la salud biopsicosocial y la resiliencia donde Clemente y Flores (2018) manifestaron que las personas más resilientes son quienes experimentan menor ansiedad ante la muerte.

Se afirma, por parte de Domínguez, López y Blanco (2017) que, existe una probabilidad más elevada de ansiedad ante la muerte en adultos mayores de edad superior a 75 años, viudos, de estudios primarios, que han sufrido pérdida familiar, y han padecido alguna enfermedad importante. Algo similar ocurre en la investigación realizada por Mohammadpour et al. (2018), quienes refieren que la ansiedad por muerte tiene una relación significativa con el estado civil de las PAM y la presencia de un compañero.

Cabe destacar las aportaciones de, Berivan, Karadag y Yıldız (2019) que tuvieron como objetivo identificar la relación entre la orientación religiosa y la ansiedad por la muerte en personas mayores de 65 años o más, resultando que las personas que tenían una alta orientación religiosa también reflejaban ansiedad por la muerte, y ésta era mayor en aquellos que vivían con sus hijos. Así mismo, diversos estudios han mostrado evidencia de que la ansiedad ante la muerte presenta diferencias de género, evidenciando que las mujeres suelen presentar mayor temor por la muerte de sus seres queridos a diferencia de los hombres lo cual se correlaciona a una mala salud física, disminución de la autoestima y falta de propósito de vida (Missler et al., 2012).

En una dirección más concreta Zhang et al. (2019) exploraron las opciones para contrarrestar la ansiedad ante la muerte en las PAM y evidenciaron que la autoestima funge como un buen mediador que influye en el significado de la vida y la muerte tomando como punto de partida los valores con los que cuenta y el sentido de existencia.

Queda clara la variabilidad en los resultados respecto a los factores influyentes y de soporte asociados al tema de la muerte y la presencia de miedo y ansiedad; sustentando la diversidad humana y las respuestas propias de cada individuo. Por ello se considera de gran importancia reflexionar sobre las intervenciones de enfermería claves aplicables en este tipo de problemática. En este sentido, Kübler-Ross (2009) plantea la opción de poder ver el tema de la muerte como algo provechoso, creativo e inclusive positivo pues su abordaje de cierta manera accede a crear una vida más reflexiva teniendo como consecuencia una existencia más comprometida y así poder llegar a un estado de maduración.

\section{Abordaje de enfermería ante el miedo y ansiedad ante la muerte}

Las intervenciones de enfermería sobre el miedo y ansiedad ante la muerte, permitirán superar los temores y coadyuvarán en generar un individuo con ganas de emprender cosas nuevas, con actitud positiva, con ímpetu de disfrutar completamente la vida y organizar efectivamente su tiempo lo cual se asocia con la presencia de un buen estado de salud (Vilches, 2000). Y es en este contexto donde la acción preventiva muestra notabilidad pues crea la acción de reconocernos y ejercer el cuidado a nuestra persona con el propósito de superar los miedos infundados que en ausencia de una intervención eficaz expondrían a la persona a evitar y no comprender el tema de la muerte como un proceso natural (Morales et al., 2011).

En realidad, no se trata de integrar una educación hacia el tema de la muerte de características lúgubres, sino más bien, las intervenciones clave radican en encaminar a las PAM a la construcción y mantenimiento de una vida con sentido, reforzada por los valores personales 
y de cierta manera motivar la participación conjunta tanto de los diferentes profesionales de la salud, como de la comunidad y la familia, puesto que en situaciones de decadencia de la salud, pérdidas o cambios importantes en la vida de las PAM, estas cuentan con un papel realmente fundamental de apoyo y orientación (Meza, 2011).

En efecto, con el apoyo adecuado se podrá efectuar en las PAM una comprensión y reorganización de las percepciones respecto a la muerte, abogando por la construcción de un autoconcepto estable y satisfactorio para que logre percibir de una manera más serena su propia muerte, reconociéndola como parte de la vida, pues la manera en que las PAM se dispongan frente a la mortalidad ciertamente definirá su proceso de muerte (Pérez, 2019).

Según Viguera (2005), citado por Tomás-sábado en 2016, manifiesta un escenario donde se puedan implementar diversas estrategias referentes a superar o controlar el miedo y la ansiedad ante la muerte denotando la importancia de la expresión de los temores a base de la escritura o con medidas alternas para modificar la percepción y/o apreciación de los mitos existentes en torno a la muerte y junto a ello el contar con el respaldo de grupos de apoyo de características semejantes en los cuales al establecerse relaciones mostrarán su eficacia motivándolos emocionalmente.

Así pues, la estrategia principal radicará en establecer y concientizar sobre la idea de generar un proyecto de vida con sentido. Aprender a detectar los elementos que contribuyen a la concepción de que la vida vale la pena, como lo son los valores éticos y espirituales. Ciertamente, cuando la vida está dirigida e inspirada por buenos valores (amor, solidaridad, responsabilidad, honradez, etc.), ejercicio ciudadano y la búsqueda espiritual, el pensamiento de la muerte no será motivo de angustia e inclusive al crear una vida con sentido permitirá en el individuo una dirección trascendente de la existencia (Villa, 2011).

Direccionado lo anterior al campo de acción de la profesión de enfermería y tomando como referencia que el sistema de atención en salud se ha visto de cierta manera fragmentado y poco integral poniendo en evidencia la limitada promoción del bienestar y atención a las necesidades emocionales y sociales relacionadas a las percepciones, batallas y sufrimientos por los que pasa el individuo (Hines, 2017), es realmente importante que enfermería intervenga en la concientización del reconocimiento personal y enseñanza de los métodos que pueden mitigar los temores infundados respecto a la muerte lo cual representa un desgaste emocional especialmente en las PAM. Esto se asocia a brindar calidad en la atención, incorporando competencias de excelencia profesional y hacer notar la autonomía del ejercicio (Luengo, Paravic y Burgos, 2017).

En este sentido, la incorporación de diagnósticos, resultados e intervenciones de enfermería es fundamental pues evoca el profesionalismo y el fundamento científico. Respecto a la temática de interés la NANDA en 2007 estableció el diagnóstico de Ansiedad ante la muerte (código 00147) el cual funge como punto de partida y análisis de las intervenciones de enfermería. Este diagnóstico está ubicado en el patrón de autopercepción-autoconcepto y hace mención de los factores relacionados que como ya se mencionó pueden ser muy variados y las características definitorias que se pueden asociar a la presencia de ansiedad ante la muerte. En este contexto el resultado esperado se centrará en que la persona reconozca, inicie y/o mantenga un estado de comodidad psicoespiritual (código 2011) manifiesto por la serenidad conexa al bienestar emocional, significado, inspiración y objetivo de la propia vida (NOC, 2018), así mismo para 
conseguir el resultado antes referido se deben plantear intervenciones de enfermería que disminuyan la ansiedad y miedo ante la muerte (Ver Figura 1).

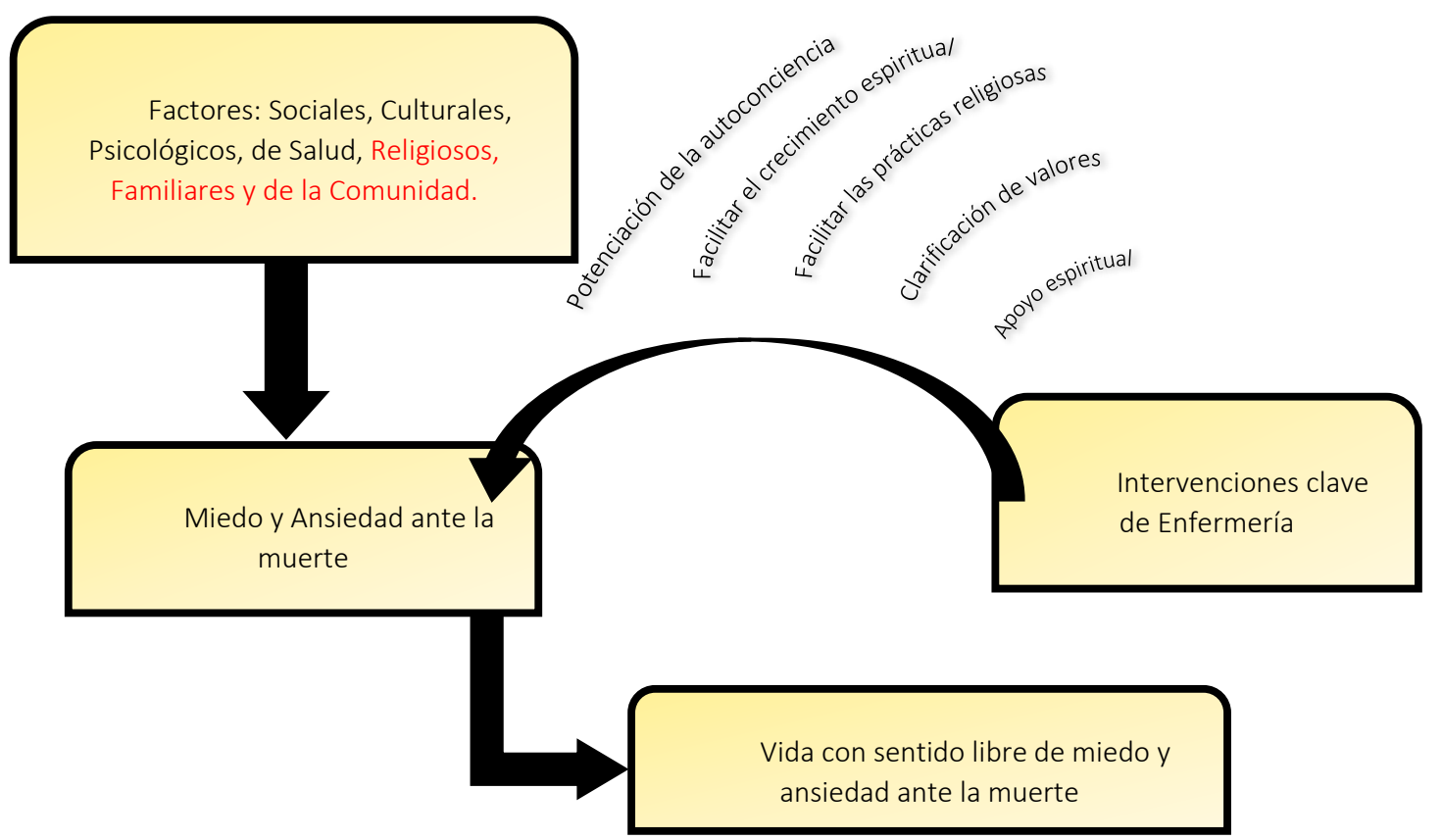

Figura 1. Relación e interacción de factores e intervenciones clave de enfermería en el contexto de miedo y ansiedad ante la muerte. Elaboración propia.

Primeramente, nos basaremos en la idea de que la persona genere un proyecto de vida con sentido con la intervención de enfermería correspondiente a la Potenciación de la autoconciencia (código 5390) referida como la ayuda que se le brinda al paciente para que explore y entienda sus pensamientos, motivaciones, sentimientos y conductas (NIC, 2018) con la acción enfermera de ayudar a las PAM a identificar sus maneras de respuesta habituales ante diferentes situaciones, orientarlo a identificar sus prioridades en la etapa de la vejez y entender cómo podría impactar la presencia de enfermedades y/o dependencias en el transcurso de la vida.

Como segundo enfoque se aborda lo referente a los valores éticos que corresponde a la intervención de Clarificación de valores (código 5480) definido como la ayuda al individuo a esclarecer sus propios valores con el objetivo de facilitar la de decisiones eficaces (NIC, 2018). Se implementará la acción de alentar al paciente a hacer una lista de valores positivos que podrán servir de guía referente a la conducta en diversas situaciones, así mismo, se puede comenzar con el planteamiento de preguntas específicas que ayuden a la PAM a reflexionar sobre el tema de la muerte o alguna situación en específico, siempre respetando y teniendo muy en cuenta los componentes individuales, éticos y legales, es decir ayudarlo a que detecte lo que es importante en la vida y priorizar los valores.

Otra de las intervenciones referente a los valores espirituales que empatan con la intervención de enfermería es el Apoyo espiritual (código 5420) definida como la acción de brindar soporte al individuo a sentir equilibrio y bienestar con un poder supremo-sobrenatural (NIC, 2018), aspecto que comúnmente se ve relegado. Importante no olvidar que forma parte de la atención holística por parte de enfermería. Se tiene como punto de partida en las acciones el 
tratar a las PAM con dignidad, respetando en todo momento sus elecciones religiosas-espirituales y si se da el caso se puede compartir la propia perspectiva espiritual, así como escuchar atentamente la expresión del adulto mayor y facilitarle el espacio de privacidad y tranquilidad para actividades según su necesidad espiritual.

Así mismo el Facilitar el crecimiento espiritual (código 5426), permitirá un desarrollo de la capacidad del paciente para identificar, comunicarse y evocar la fuente de significado, propósito, consuelo, fuerza y esperanza en su vida (NIC,2018). Lo anterior fomenta que la espiritualidad en la persona adulta mayor tenga una comprensión del acto mismo que hace trascender en sus dimensiones de vida, apropiándose de un crecimiento y autodescubrimiento espiritual que le permite al ser avanzar en la comprensión de la vida, de su vida, resignificando y avanzando a medida que la asunción del espíritu es mayor en los ambientes de actuación de cada persona (Palacios, 2015).

Finalmente, una de las intervenciones plasmadas que permite afrontar el miedo y ansiedad ante la muerte es el Facilitar las Prácticas Religiosas (código 5424) el cual se fomenta el uso y la participación en cualquier ritual o práctica que no perjudique la salud, mediante la gestión de servicios religiosos, así como dar libertad para expresar sus sistemas de creencias y visiones del mundo basado en el respeto (NIC,2018). Estas prácticas religiosas pueden incluso reajustar el significado de los problemas de la vida diaria para que éstos parezcan más manejables, aumentando así los niveles generales de satisfacción y sentido con la vida, es por ello que más que la actividad religiosa en sí, es la fe personal la que parece ayudar a las personas adultas mayores a enfrentar el estrés y el temor a la muerte (Salgado, 2014; Oñate et al., 2018).

Es así que se plantea la situación lógica de contribuir como profesionales de la salud a satisfacer las demandas que presenten los individuos, en concreto las PAM, quienes en base a sus vivencias, experiencias y sabiduría podrán llegar a un estado de conocimiento sobre el fenómeno de la muerte asociándolo a un hecho real, indiscutible y seguro de que se presente en cualquier momento y en cualquier situación y tener como consecuencia la prevención o reducción del miedo y ansiedad que se pudiera presentar.

\section{Conclusión}

Al establecer un enfoque en los cambios y pérdidas que se hacen aún más evidentes y significativas en la etapa de la vejez, prácticamente se estaría visualizando un amplio panorama de necesidades expresadas o inexpresadas por las PAM las cuales al sentirse cerca del fin de su vida sin duda alguna requieren de un cuidado sostenido y pleno que cubra sus necesidades psicosociales y emocionales situación de la que puede ser participe el profesional de enfermería para obtener como resultado en el individuo, una vida más participativa, responsable, reflexiva y consciente de sí mismo (Villa, 2011).

Efectivamente, conforme las personas van envejeciendo va integrando en su conciencia aspectos sobre su mortalidad, sin embargo, al ser la muerte un tema tan abstracto no todos los individuos aceptan hablar sobre ello sumado a que comúnmente la sociedad tiende a esconder o simplemente restarle importancia optando por disfrazarla con conceptualizaciones alejadas de entenderla como un proceso natural (Sugar, 2019). Es en este punto donde se pueden 
implementar las estrategias de acción y educación para disminuir los temores infundados y aceptar la mortalidad de manera consciente y tranquila (Sánchez \& Salas, 2015).

Es por ello que la preparación de las PAM sobre el tema del fin de la vida definitivamente puede favorecer a que se pueda crear una nueva perspectiva sobre la vida y fomentar el deseo de revalorar las situaciones y como consiguiente tomar decisiones más sólidas cargadas de sentido y beneficio. Así mismo, como se ha expuesto, el disminuir el miedo y la ansiedad por la muerte en las PAM puede desempeñar un papel importante en la mejora de su salud mental y calidad de la vida (Zhang et al., 2019).

En este caso, el profesional de enfermería que destaca por la relación tan estrecha con el individuo, cuenta con las herramientas científicas necesarias para poder intervenir de manera eficaz en el cuidado y educación de las PAM respecto a la manera en cómo se perciben así mismos, al trayecto de su vida y culminación de la misma. Es fundamental que se logren detectar las necesidades de cuidado de una manera integral que reconozca al individuo como un todo que atienda tanto el aspecto físico, biológico como el emocional y espiritual.

Es importante concientizarse sobre la capacidad y compromiso adquirido en el ejercicio profesional, incorporando estrategias de cuidado, actualizando el conocimiento y brindando un cuidado de calidad dirigido en este caso a las PAM quienes sin duda alguna se enfrentan a una serie de cambios, desconciertos, incertidumbres y temores ya sea por su situación de salud o simplemente por la noción del paso del tiempo. Esto implica ofrecer un cuidado centrado en la persona con toda su realidad humana integrando el sentido de la vida, así como el abordaje de la muerte de una forma que no impacte negativamente su bienestar general como ser humano.

En definitiva, el considerar la aplicación de las intervenciones claves de enfermería direccionadas a la atención de los temores y ansiedad ante la muerte que experimentan las PAM, brindará un acceso hacia una comprensión sobre el final de la vida como un hecho natural adquiriendo a la vez una perspectiva más consciente del propio ser y todo alrededor de él.

\section{Referencias}

Álvarez, L.Y. y Flórez G.Z. (2008). Revista Colombiana de Psicología, Actitudes hacia la muerte en un grupo de adultos intermedios y mayores con enfermedad crónica (hipertensión, diabetes y cáncer) en la ciudad de Bucaramanga, (17), 75-82.

Berivan, B. A., Karadag, A.S., Yıldız, M. (2019). Relationship Between Religious Orientation and Death Anxiety in Elderly Individuals, Journal of Religion and Health, doi.org/10.1007/s10943-019-00917-4.

Brudeka, P. \& Sekowskib, M. (2019). Wisdom as the mediator in the relationships between religious meaning system and attitude toward death among older adults, Death Studies, doi.org/10.1080/07481187.2019.1609136.

Carmelo, A. \& Comas L. (2014). Miedos, adaptabilidad y evolución. En ¿Existe la muerte? (pp. 915). Barcelona: Plataforma.

Cartay, R. (2002). La Muerte. Fermentum Revista Venezolana de Sociología y Antropología, 12(34), 447-470. 
Ceberio, M. R. (2013). El último tramo. Cuarta edad, duelos y muerte. En El cielo puede esperar. La cuarta edad: Ser anciano en el siglo XXI (pp. 248-270). Madrid: Morata.

Domínguez, A. J., López, C. A. \& Blanco L. A. (2017). Evaluación de la ansiedad ante la muerte en adultos mayores ourensanos. Revista de estudios e investigación en psicología y educación. doi: https://doi.org/10.17979/reipe.2017.0.14.2471

Duran, B. T., Maldonado, V. M. A., Martínez, A. M., Gutiérrez, S. G., Ávila, A. H., López, G. S. (2020). Miedo ante la muerte y calidad de vida en adultos mayores, Enfermería Global, doi.org/10.6018/eglobal.364291.

Clemente, G. T. \& Flores, R. N. (2018). Ansiedad, miedo y actitudes hacia la muerte en población general mayor de edad: evaluación y repercusión en variables psicológicas y de salud. Recuperado de: https://gredos.usal.es/handle/10366/137927

Hines, M. E. (2017). Turning Words Into Action Advancing the Role of Advanced Holistic Nurses. Journal of Holistic Nursing, 35(4), 316 -317.

Kübler-Ross, E. (2009). Why Is It So Hard to Die?. En Death: The Final Stage (pp. 32-38). New York: Touchstone.

Luengo, M. C., Paravic, K. T. \& Burgos, M. M. (2017). Profesionalismo en enfermería: una revisión de la literatura. Enfermería Universitaria, 14(2), 131-142.

Meza, R. J. (2011). Introducción. En La muerte: Siete visiones, una realidad (pp. 13-15). Bogotá, Colombia: Pontificia Universidad Javeriana.

Missler, M., Stroebe, M., Geurtsen, L., Mastenbroek, M., Chmoun, S., Houwen, K. (2012). Exploring death anxiety among elderly people: a literature review and empirical investigation. Omega, 64(4), 357-379.

Mohammadpour, A., Sadeghmoghmoghadam, L., Shareinia, H., Jahani, S. \& Amiri, F. (2018). Investigating the role of perception of aging and associated factors in death anxiety among the elderly. Clinical Interventions in Aging, 13, 405-410.

Morales, F. M., Quintero, S. M. \& Pérez, G. R. (2011). La educación tanatológica para el bienestar de la salud. Revista digital Universitaria, 12(2). Recuperado de http://www.revista.unam.mx/vol.12/num2/art17/art17.pdf

Navas, O. W. \& Vargas, M. J. (2012)., Trastornos de ansiedad: revisión dirigida para atención primaria. Revista Médica de Costa Rica y Centroamérica, 69(604), 497-507.

North American Nursing Diagnosis Association (NANDA), (2018-2020). Diagnósticos Enfermeros definiciones y clasificación (pp. 354). Barcelona, España: Elsevier.

Nursing Interventions Classification (NIC), (2018-2020). Clasificación de Intervenciones de Enfermería (pp. 80, 107, 208, 213, 357). Barcelona, España: Elsevier.

Nursing Outcomes Classification (NOC), (2018-2020). Clasificación de Resultados de Enfermería, Medición de Resultados en Salud (pp. 376). Barcelona, España: Elsevier. 
Ochoa, V. J., Cruz, O. M., Pérez, R. M., Cuevas, G. C. E. (2018). El envejecimiento: una mirada a la transición demográfica y sus implicaciones para el cuidado de la salud. Revista de Enfermería del Instituto Mexicano del Seguro Social, 26(4), 273-280.

Oñate, Ma. E., Mesurado, B., Rodríguez, L. M., Moreno, J. E. (2018). Práctica religiosa y sentido de vida en adultos jóvenes. Revista de Psicología, 14(27). Recuperado de:

http://bibliotecadigital.uca.edu.ar/greenstone/cgi-bin/library.cgi?a=d\&c=Revistas\&d=practicareligiosa-sentido-vida

Organización Mundial de la Salud. (2018). Notas descriptivas, Envejecimiento y Salud. Recuperado de: https://www.who.int/es/news-room/factsheets/detail/envejecimiento-y-salud

Palacios, V.C. (2015). La espiritualidad como medio de desarrollo humano. Cuestiones Teológicas, 42(98), 459-481.

Pérez, M. D. (2019). Percepción de la muerte a través de la cultura en los adultos mayores de la comunidad de San Pablo Tijaltepec, Tlaxiaco, en Oaxaca (artículo de opinión). Recuperado de: $\quad$ https://revistaaletheia.ieu.edu.mx/documentos/A_opinion/2019/8_Agosto/Art_Op_3.pdf

Restrepo, M. E. (2013). Miedo a la muerte, afrontamiento, religiosidad y salud, en población romani/gitana de SES medio-bajo (tesis doctoral). Recuperado de: https://riuma.uma.es/xmlui/handle/10630/6969

Rivarola, R. L. (2016). Temor hacia la muerte, espiritualidad y florecimiento en adultos mayores de lima metropolitana (tesis). Recuperado de: http://tesis.pucp.edu.pe/repositorio/handle/20.500.12404/7710

Salgado, A. (2014). Revisión de estudios empíricos sobre el impacto de la religión, religiosidad y espiritualidad como factores protectores. Propósitos y Representaciones, 2(1), 121-159. doi: http://dx.doi.org/10.20511/pyr2014.v2n1.55

Sánchez, J. M., \& Salas, G. V. (2015). Actitud ante la muerte y el proceso de morir, propio y el de los demás (Tesina). Recuperada de http://www.tanatologiaamtac.com/descargas/tesinas/286\%20actitud.pdf

Sandrin, L. (2013). El camino del morir. En Cuidar al enfermo. (pp. 39-41). España: DESCLEE DE BROUWER.

Sugar, J. A. (2019). Death, dying, and bereavement. En Introduction to Aging. (pp. 259-264). New York: Springer Publishing Company.

Tomás-sábado, J. (2016). Introducción, La ansiedad, La ansiedad ante la muerte. En Miedo y ansiedad ante la muerte: Aproximación conceptual, factores relacionados e instrumentos de evaluación. (pp.1, 9-41, 116). Barcelona, España: Herder.

Uribe, R. A. y Valderrama, O.L., López, S. (2007). Actitud y miedo ante la muerte en adultos mayores. Pensamiento Psicológico, 3 (8), 109-120. 
Vilches, L. (2000). Concepciones, creencias y sentimientos acerca de la muerte en adultos mayores de nivel educación superior. Revista de Psicología de la Universidad de Chile, 9 (1).

Villa, P. E. (2011). Muerte y cultura. En La muerte: Siete visiones, una realidad (pp. 64-105). Bogotá, Colombia: Pontificia Universidad Javeriana.

Zhang, J., Peng, J., Gao, P., Huang, H., Cao, Y., Zheng, L., Miao, D. (2019). Relationship between meaning in life and death anxiety in the elderly: self-esteem as a mediator, BMC Geriatrics, doi.org/10.1186/s12877-019-1316-7. 